\title{
Pertinencia de las tecnologías de la información y la comunicación para el fortalecimiento de la comprensión lectora ${ }^{1}$ \\ Relevance of information and communication technologies (ICT) for the strengthening of reading comprehension
}

DOI: http://dx.doi.org/10.17981/cultedusoc.9.3.2018.71

Artículo de investigación. Fecha de recepción: 15/06/2018. Fecha de aceptación: 27/11/2018

\author{
Glen Jiménez Ariaz²; \\ Wuilian Ortiz Severiche; Eucaris Pérez Gómez; \\ Eduardo Romo Boneth y Luis Crespo Arévalo ${ }^{3}$
}

IED Rural de Media Luna, sede Erm Manuel de Jesús Escorcia, Pivijay-Magdalena. (Colombia)

glen070880@hotmail.com

Para citar este artículo:

Jiménez, G.,Ortiz, W., Pérez, E., Romo, E. y Crespo, L. (2018). Pertinencia de las tecnologías de la información y la comunicación para el fortalecimiento de la comprensión lectora. Cultura. Educación y Sociedad 9(3), 603-612. DOI: http://dx.doi.org/10.17981/cultedusoc.9.3.2018.71

\section{Resumen}

La integración de las tecnologías de la información y comunicación (TIC) en los procesos educativos resulta importante por cuanto dinamiza la relación docente - estudiante. Al respecto, el presente artículo se orienta al análisis de la pertinencia de los objetos virtuales de aprendizaje en el proceso de mediación didáctica. El diseño metodológico es de tipo mixto. Se inicia a partir de un proceso descriptivo y reflexivo de los resultados de la identificación de las prácticas pedagógicas docentes que se trabajan de forma tradicional en la Institución Educativa ERM Manuel de Jesús Escorcia. La población de estudio corresponde a estudiantes del grado $5^{\circ}$. Se aplicaron instrumentos de recolección de información a docentes para conocer sus percepciones con la temática estudiada. Los resultados indicaron que la estrategia con utilización de las TIC, mejoró la comprensión lectora en los estudiantes. Como conclusiones tenemos, la importancia que tiene, que los docentes diseñen estrategias didáctica-pedagógica, dejando abierta la posibilidad de ser utilizada en otras áreas del saber.

Palabras clave: tecnologías de la información y comunicación, acto pedagógico, comprensión lectora.

\section{Abstract}

The integration of information and communication technologies (ICT) in educational processes is important because it stimulates the teacher-student relationship. In this regard, this article is oriented to the analysis of the relevance of virtual learning objects in the process of didactic mediation. The methodological design is of a mixed type. It starts from a descriptive and reflective process of the results of the identification of teaching pedagogical practices that are worked in a traditional way in the Educational Institution ERM Manuel de Jesus Escorcia. The study population corresponds to 5th grade students. Data collection instruments were applied to teachers to know their perceptions with the subject studied. The results indicated that the strategy with the use of ICTs improved reading comprehension in students. As conclusions we have, the importance that has, that teachers design didactic-pedagogical strategies, leaving open the possibility of being used in other areas of knowledge.

Keywords: information and communication technologies, pedagogical act, reading comprehension.

1 Este artículo ha sido derivado del Programa de Fortalecimiento de la Cultura Ciudadana y Democrática CT+I a través de la IEP apoyada en TIC en el Departamento de Magdalena: CICLON.

2 Lider grupo de investigación "Inedemistas de Avianca"

3 IED Rural de Media Luna. Sede Erm Manuel De Jesús Escorcia, grupo de investigación inedemistas de Avianca. Pivijay, Magdalena.

- The author; licensee Universidad de la Costa - CUC.

Cultura, Educación y Sociedad vol. 9 no. 3, pp. 603-612. Diciembre, 2018

Barranquilla. ISSN 2389-7724 Online 


\section{Introducción}

Este estudio se realizó en Avianca, corregimiento ubicado en el municipio de Pivijay, localizado al noroccidente del departamento del Magdalena. En una población muy vulnerada económica y políticamente, con escasos recursos económicos, que ha sido afectada por conflictos y amenazas de grupos armados; hecho éste del que está comenzando a recuperarse de enfrentamientos internos con la guerrilla. En este período posconflicto, la comunidad está buscando salir de una historia cargada de violaciones a sus derechos humanos y desplazamientos forzados de sus integrantes.

La institución educativa ERM Manuel de Jesús Escorcia ofrece estudios hasta octavo de secundaria con una población relativamente pequeña (160 estudiantes), se encuentra actualmente sumida en una problemática de carácter académico, debido a los malos hábitos de estudio y bajo rendimiento. Es característica notoria la situación de apatía de los estudiantes por la lectura; entre los factores que pueden estar afectando e influyendo en la problemática, se considera que la baja escolaridad de los padres de familia, la inexistencia de bibliotecas y libros para los estudiantes, quienes además viven en una zona alejada del acceso a estos recursos, con los cuales puedan desarrollar actividades académicas desde sus casas, y que al no poder hacerlo genera en los estudiantes un desapego y desistieres por el estudio.

Por otro lado, no es menos importante tener en cuenta que desde las prácticas docentes tradicionales, los estudiantes no encuentran contenidos y o elementos pedagógicos atrayentes y motivantes, que generen un interés por aprender y apropiarse del conocimiento; cabe resaltar que los aspectos nutricionales y las condiciones socioeconómicas, como el conflicto armado o el condicionamiento de víctima del desplazamiento, son variables de alta complejidad que afecta el éxito en el acto pedagógico (Avendaño-
Villa, Cortés-Peña, y Guerrero-Cuentas, 2015), (Herrera, 2016).

Por ende, si se reconoce el constante avance de las tecnologías de la información y comunicación (TIC), y de sus herramientas de apoyo para el mejoramiento de la práctica educativa, surge esta iniciativa de busca cubrir la necesidad de ejecutar un proyecto que permita implementar las TIC como herramienta de aprendizaje desde la práctica pedagógica.

Las TIC como herramientas al acto pedagógico docente, desde la didáctica, brindan beneficios al ser capaces de captar el interés de sus estudiantes, debido a que a través de estas herramientas se abren las posibilidades de comunicación y por lo tanto fortalecen la relación docente-estudiante, direccionando al mejoramiento del trabajo escolar, y en este sentido, la educación cumple un papel importante, puesto que es concebida como promotora de valores compartidos y asume responsabilidad en el desarrollo creativo y afectivo de los educandos (UNESCO, 2008).

Los procesos de enseñanza aprendizaje, pueden ser fortalecidos y dinamizados a través de los Objetos Virtuales de Aprendizaje (OVA), que son herramientas que organizan la experiencia que va a vivir el alumno, con intenciones claras, son utilizadas en programas virtuales; pero también son aprovechables en la modalidad presencial, para darle un carácter innovador a la cotidianidad de situaciones del aula.

Entre los lineamientos sobre la inserción de las TIC en los primeros grados de la educación, el estado colombiano ha señalado un conjunto de ventajas para la aplicación de los OVA (PoliVirtual in Colombia, 2016, p. 1):

- "Esta herramienta digital le sirve al tutor como una extensión para conocer el avance del estudiante en los temas involucrados y allí puede monitorear qué tan importante y viable fue el material para los alumnos. 
- El tutor virtual o el docente, puede usar, reutilizar y actualizar constantemente los documentos e información que integre en las OVA que utilice durante sus clases.

- Las OVA se puede adaptar a cualquier plataforma LMS o plataformas de educación virtual.

- Garantiza el uso efectivo de las Tecnologías de Información y Comunicación (TIC), tanto para tutores como para alumnos.

- Le facilita la búsqueda a los estudiantes de los materiales que utilicen durante su experiencia virtual y uso de plataformas de aprendizaje"

El manejo de esta alternativa, por ser una innovación, hace requerimientos importantes a la formación y dedicación de los docentes y a las condiciones de uso de las tecnologías en la escuela y en la comunidad. Todos esos desafíos se esperan trabajar en esta investigación.

La población de estudio corresponde a estudiantes del grado $5^{\circ}$ de la ERM Manuel de Jesús Escorcia, los cuales se distribuyen en edades promedio de 10 años, pertenecientes al Municipio de Pivijay, corregimiento de Avianca y a los rededores del Municipio de Pivijay. Además, del cuerpo docente de la institución educativa conformado por 8 maestros que manejan las diferentes áreas y las asignaturas del ciclo básico y medio.

A nivel internacional, se hace referencia al uso de herramientas tecnológicas como elemento de apoyo para el desarrollo de habilidades y/o comprensión de un tema para estudiantes de básica y media, como lo presenta Arroyo, et al. (2010), quienes describen un estudio de innovación educativa, que se planteó el objetivo de desarrollar la comprensión lectora a través de experiencias que se promueven transversalmente en las asignaturas de Ciencias, Español y Educación en los grados de segundo y tercero de educación secundaria.
La innovación consistió en desarrolle una práctica pedagógica que fuera de carácter motivante, significativa y colaborativa para los estudiantes, utilizando como apoyo las TIC y promoviendo el hábito de lectura y el mejoramiento de competencias comunicacionales, tan importante para el proceso formativo e integral de los estudiantes.

Como lo describe Escobar (2016), las necesidades comunicativas de los seres humanos permiten los adelantos tecnológicos, siendo el uso de las TIC muy importante, ya que, las instituciones de educación en especial, las de educación superior, requieran la implementación de las ova en sus clases.

Por otro lado como lo define (Clavijo, et al 2015), una de las herramientas que nos ofrece las TIC, son los Objetos Virtuales de Aprendizaje (OVAS), en el que un objeto virtual de aprendizaje es un conjunto de recursos digitales, auto contenible y reutilizable, con un propósito educativo y constituido por al menos tres componentes internos: Contenidos, actividades de aprendizaje y elementos de contextualización.

Las tecnologías de la información y la comunicación son de amplia importancia para mejorar y dinamizar las actividades de aprendizaje en las escuelas, desde la realidad educativa, las TIC permiten contribuir a desarrollar competencias en el desenvolvimiento personal, educativo, social y laboral (Parra, Gómez y Pintor, 2014, p. 5.).

A nivel nacional investigaciones como la descrita por Parra, Gómez y Pintor (2014), identificaron los factores que inciden en el uso de las TIC en los procesos de enseñanza aprendizaje, en un curso de primaria de una escuela colombiana, utilizando un enfoque cualitativo, mediante obtención de información mediada por entrevistas, observaciones y revisión de documentos, la investigación permitió destacar entre sus resultados que, entre los factores que influyen se encuentra la capacitación, disponibilidad de recursos y el apoyo institucional. 
Un aporte importante que se obtuvo de este estudio fue caracterizar el desafío que le plantea la incorporación de las TIC a la práctica pedagógica y más específicamente a los docentes, y al propio proceso de aprendizaje; porque plantean necesidades sobre las cualidades que deben desarrollar los estudiantes, para descifrar los mensajes y adecuarlos a las etapas de desarrollo que atraviesan y contextualizarlos en relación con la realidad e historia vivida, para darle significado. Quiere decir que el diseño de la experiencia docente debe considerar todos esos factores que median el proceso formativo; lo cual exige competencias docentes que deben ser reaprendidas y renovadas constantemente.

Respecto al fundamento de las OVA, según (Cabrera-Medina, Sánchez-Medina y Rojas-Rojas, 2016), corresponde al uso de recursos tecnológicos, asociados a propósitos educativos y formativos, mediante la ocurrencia de experiencias significativas en las que, conforme a las necesidades del estudiante se genera aprendizajes, utilizando las tecnologías en el aula de clases y fuera de ella, entre los elementos que se pueden tener en cuenta en las redes de OVA, tenemos los siguientes: Los objetos procedentes de los medios o recursos tecnológicos, tales como fotografías y videos; los objetos construidos a partir de procesar el conocimiento, tales como esquemas, gráficos, audios grabados y hasta los mismo textos. También las herramientas aportadas por la tecnología, los simuladores, los ejercicios que se elaborar para graduar el aprendizaje y hasta los juegos didácticos. Todas estas herramientas pueden formar parte de experiencias sistematizadas en cursos, tutoriales o también organizados en módulos: los cuales pueden ser administrados en experiencias formativas en las redes, en el aula o fuera de ella. Todas estas modalidades, traen a la enseñanza posibilidades muy variadas que exigen un mayor manejo de los avances científicos y un mayor contacto con el contexto que le da sentido a esos contenidos de aprendizaje (Fandos, 2003).

Todas las temáticas deben estar actualizadas e incorporar avances informáticos, tecnológicos y científicos que exigen a los actores, docentes y alumnos, unas competencias de indagación y comprensión de la ciencia en la teoría y en la forma de llevarlo a la práctica. Lo más importante es que la experiencia esté centrada en lograr el aprendizaje del alumno, en lo personal y en lo colectivo, en el conocimiento y en su aplicación (Cabrera, et al. 2016)

El desarrollo local de las OVA, requiere de una conceptualización y estructuración en Colombia y el mundo, las instituciones de Colombia han dirigido en importante medida sus esfuerzos para incluir los procesos que se relacionen con ellas, dentro de sus programas educativos (Cabrera, et al 2016).

Teniendo en cuenta los elementos anteriormente descritos, a partir de la práctica docente en la institución educativa se puede tener en cuenta que para la aplicación de los objetos virtuales de aprendizaje se requiere un manejo conceptual y práctico del uso de las herramientas tecnológicas por parte del personal docente (Vélaz y Vaillant, 2009).

Desde las estrategias que se plantean en la utilización de las TIC, y de la disposición de estas herramientas a los estudiantes, desarrollan búsquedas por internet entorno a intereses del desarrollo de la clase acorde como se defina el tema, desde esta herramienta se mantiene una conectividad a internet desde donde pude descargar contenidos en tiempo real, como elementos de insumo y referencia en el desarrollo de la clase, mientras el docente con el apoyo de material audiovisual como televisor y o vídeo beam, realiza la presentación de cuadros, videos, e información multimedia que contiene los elementos conceptuales, de contenido y explicación del tema de la clase. 
Convirtiéndose en este sentido en una actividad completamente dinámica, en la que el estudiante se convierte en participe activo indagador de la información y el conocimiento (Martínez y Badillo, 2013).

\section{Metodología}

El diseño metodológico se basa en una metodología de tipo mixto. Se inicia a partir de un proceso descriptivo y reflexivo de los resultados de la identificación de las prácticas pedagógicas docentes que se trabajan de forma tradicional en la Institución Educativa. La población de estudio corresponde a estudiantes del grado $5^{\circ}$ de la ERM Manuel De Jesús Escorcia, los cuales se distribuyen en edades promedio de, 10 años.

Este trabajo de investigación se concentra en un estudio de caso, porque tiene la intención de estudiar un contexto a profundidad, en la que se ha observado problemas que deben contribuir a reflexionar alternativas para resolverlos. Tiene el propósito de replantear caminos para mejorar y actualizar la práctica educativa en lo referente al desarrollo de la comprensión lectora. Sigue un proceso mixto, cuanti-cualitativo, porque trabaja con los hechos en su cantidad y en la calidad de las relaciones que se establecen en la realidad estudiada. Se discuten y analizan los datos y se realizan inferencias para transformarla e intervenirla (Hernández Sampieri y Mendoza, 2010)..

\section{Técnicas e Instrumentos}

Según Hernández (2010), esta investigación es mixta y además es de tipo cuasi experimental. Se aplicó un pre-test, se intervino con una situación didáctica apoyada en TIC y luego se realizó un pos-test a un sólo grupo, lo cual permitió analizar la conducta de entrada y el logro de resultados en cuanto a comprensión lectora y a los resultados en la variación introducida en las estrategias y recursos de enseñanza
Los materiales y herramientas de los instrumentos tecnológicos para la implementación de las OVA son los siguientes:

- Computador portátil.

- Tablet electrónica.

- Televisor LCD.

- Paquete office básico: Microsoft Word, Microsoft Excel y Microsoft PowerPoint.

- Internet y Wi-Fi.

- Video beam.

Los anteriores elementos de equipos y herramientas corresponden a los instrumentos con los que se dispone en la institución educativa y que se requieren, con el objeto de realizar la práctica pedagógica apoyada en estos instrumentos permitiendo el desarrollo de los contenidos curriculares del plan de cada área, a partir de la utilización de ellos, como mecanismos atrayentes del interés estudiantil.

El instrumento que se aplicó a los docente, planteaba generar una identificación de las prácticas docentes que se trabajan en la institución, así como, el reconocimiento de sus habilidades en el uso de las TIC y de su implementación en el aula de clases, como se describe a en el instrumento, posteriormente se desarrolló la fase de análisis de resultados.

\section{Resultados}

El presente trabajo orientado a implementar las OVA, como herramienta de apoyo al acto pedagógico docente que fortalece el proceso de comprensión lectora, en estudiantes de la básica primaria y media permite describir el mejoramiento de la práctica pedagógica docente, en especial porque su motivo surge ante la necesidad de favorecer un mayor interés por parte de los estudiantes respecto a los hábitos de estudio.

Además, durante este proceso se pudo generar un análisis descriptivo de las prácticas educativas pedagógicas docentes 
que se asistían en la institución de forma tradicional, así como la caracterización en el conocimiento y uso de las TIC en el acto pedagógico. La materialización de la experiencia apoyada, fue posible a través de un proceso de referenciación bibliográfica, y práctica experimental en el que el docente desde la integración curricular del uso de las TIC, en los procesos de aprendizaje académicos e investigativos, permitiendo en este sentido un impacto sobre la comunidad educativa, en el que se promovió un mayor interés por aprender a partir de la inclusión de contenidos y herramientas digitales que influyeron en un cambio de la dinámica del aprendizaje ver figuras 1, 2 y 3 . Actualmente aún se requiere una mejor cualificación del cuerpo docente para poder influir en un mayor impacto sobre la población estudiantil.

A continuación, se describe los resultados descritos durante la investigación. La aplicación del instrumento permitió obtener los siguientes resultados:
Se evidencia que los docentes en su mayoría, están de acuerdo siempre en la elaboración de sus clases de acuerdo con los planes de área, y planificación de los recursos para el desarrollo de la clase. Sin embargo, la conformación de semilleros de investigación entre los estudiantes de su clase se respondió en mayor medida como "a veces".

En este se evidencia que tres de los docentes encuestados manifiestan nunca trabajar el estado de arte como estrategia, casi nunca realizan clases magistrales, dirigiéndose en mayor medida las mesas redondas las exposiciones orales y mapas conceptuales.

Las figuras 3, 4 y 5 representan el conocimiento y o utilización personal de las herramientas tecnológicas en la población docente, evidenciándose que un $29 \%$ aun no maneja el computador, y respecto a quienes si lo hacen no todos manejan los paquetes u o herramientas del paquete office básico: Microsoft Word, Microsoft Excel y Microsoft PowerPoint, con un $43 \%$ de desconocimiento.

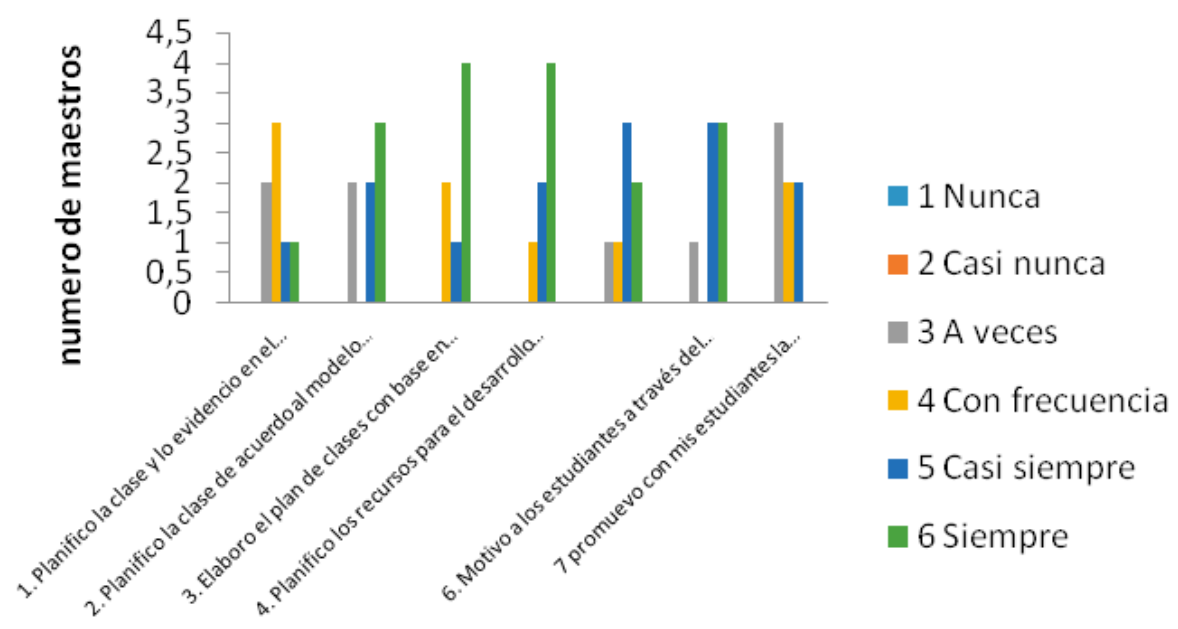

Pregunta orientadora

Figura 1. Representación gráfica de las respuestas establecidas por la población de estudio docente, se encuestaron 7 maestros correspondientes a la planta de la sede. 


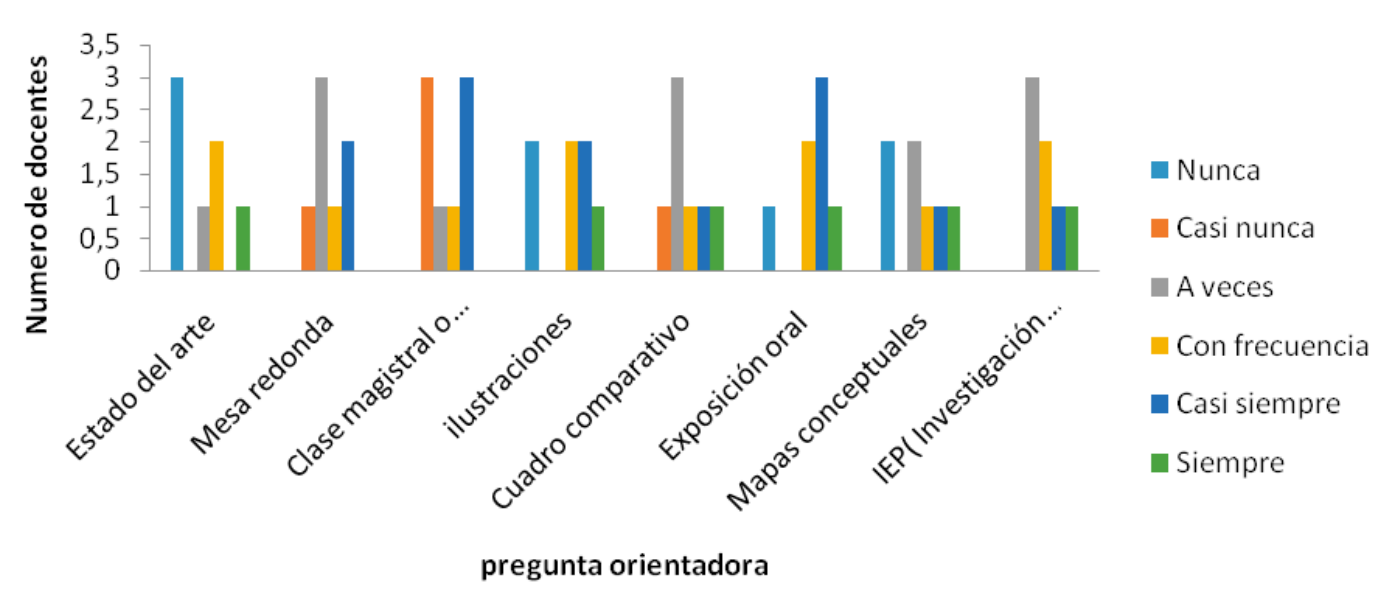

Figura 2. Representación gráfica de las Estrategias pedagógicas más comunes de implementación en las escuelas.

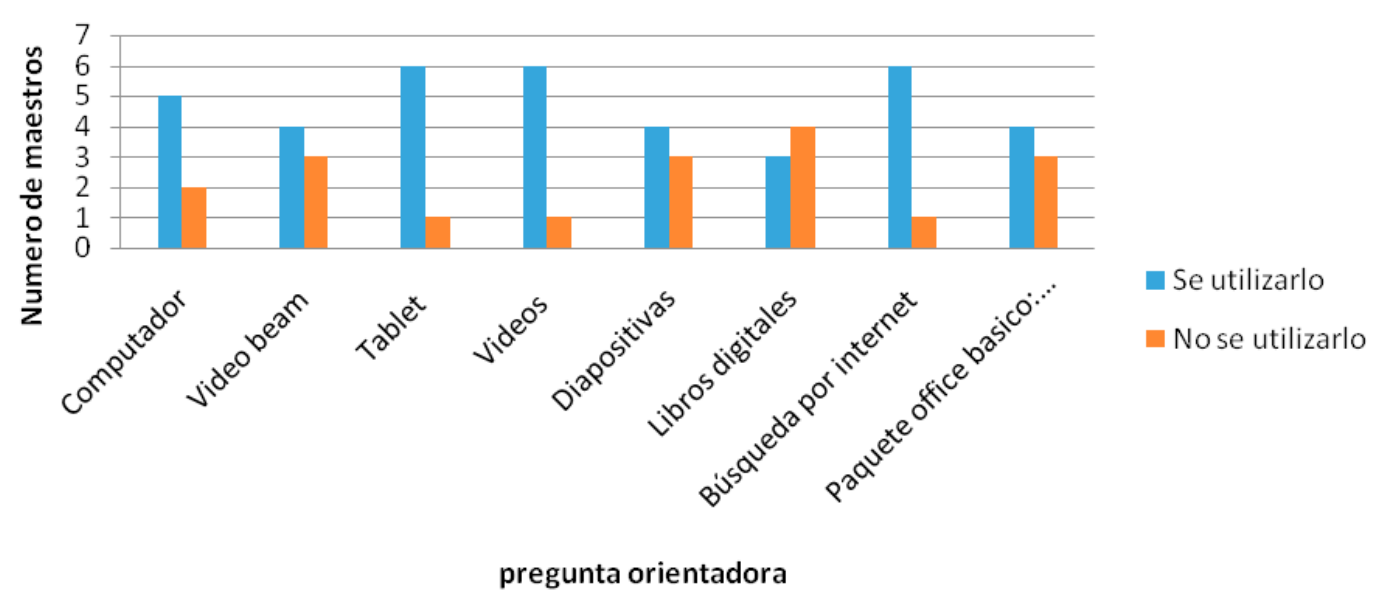

Figura 3. Representación gráfica del conocimiento relacionado a la utilización personal de los equipos electrónicos y herramientas digitales.

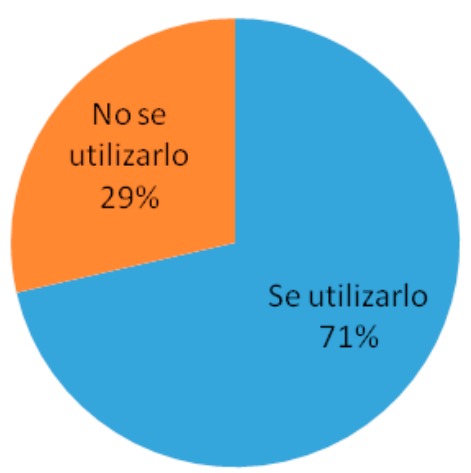

Figura 4. Relación porcentual del manejo del computador. 


\section{Paquete office basico: Microsoft Word, Microsoft Excel y Microsoft PowerPoint.}

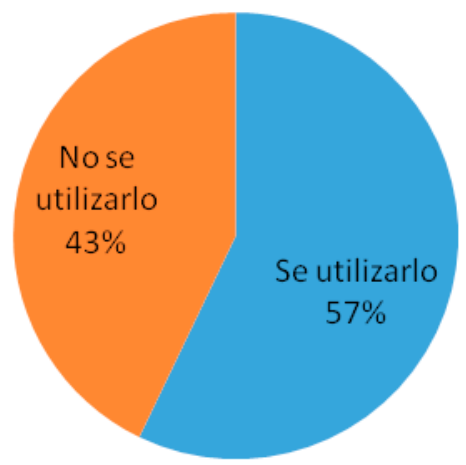

Figura 5. Relación porcentual en el manejo de los programas básicos digitales.

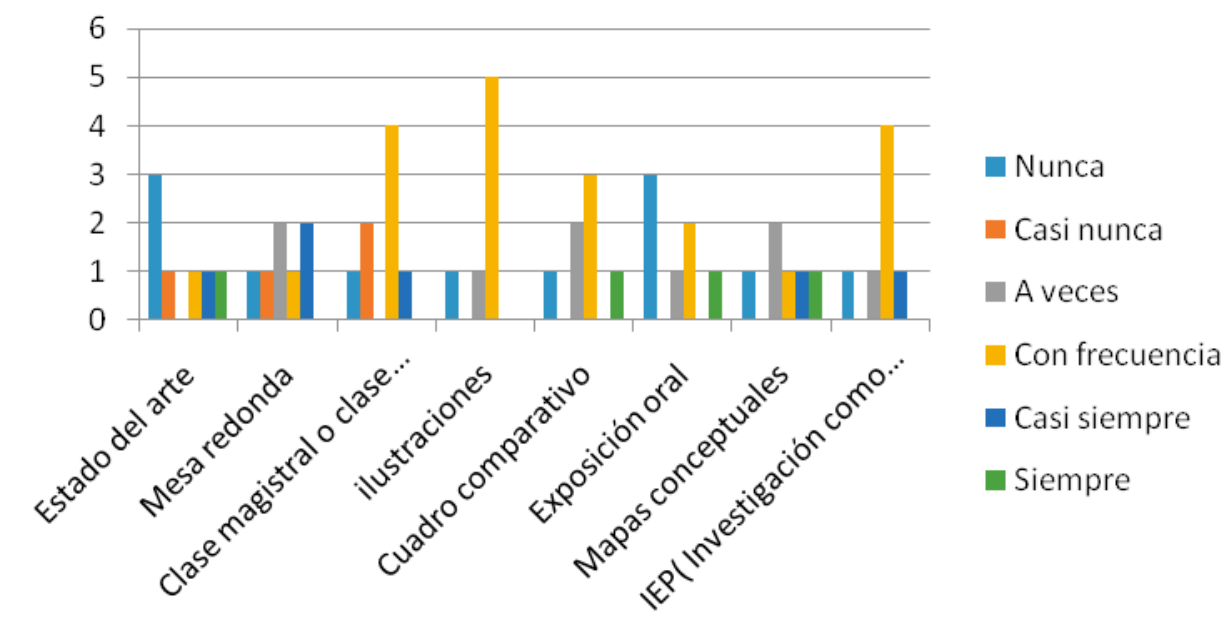

Figura 6. Representación gráfica de la aplicación de las TIC, desde las estrategias pedagógicas.
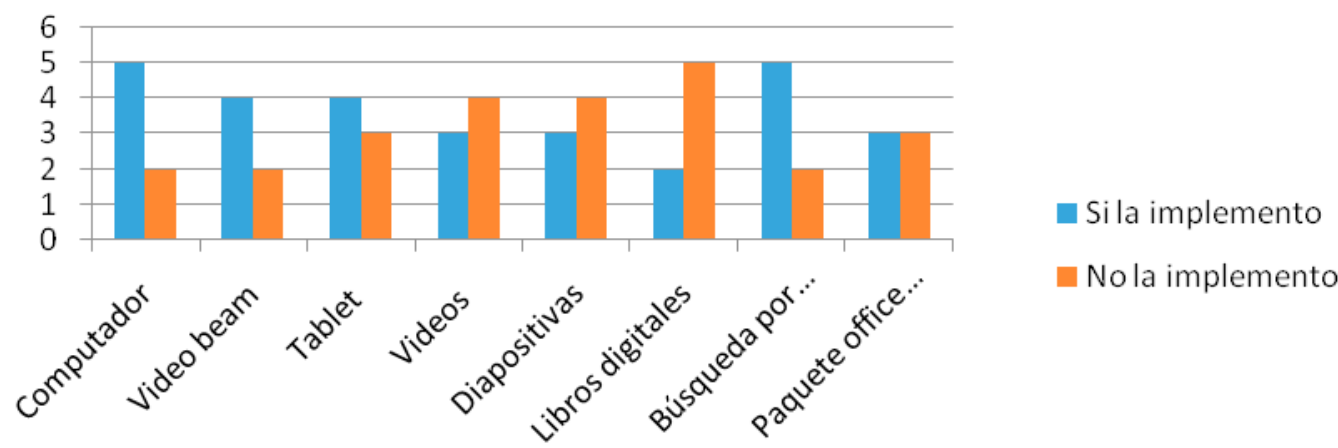

Figura 7. Representación gráfica de la implementación de las herramientas y programas digitales en las prácticas pedagógicas decentes. 
Se evidencia la aplicación de las TIC que usan los docentes desde sus prácticas pedagógicas, siendo mayormente frecuente el uso de estas entre las clases magistrales, ilustraciones e investigación como estrategia pedagógica, con una menor frecuencia de uso en los mapas conceptuales, estado del arte y exposiciones orales.

De acuerdo con estos resultados, el mayor uso del computador, video beam, y Tablet como herramienta de apoyo al acto pedagógico. Sin embargo, el uso de libros digitales, diapositivas y el paquete office son en menor medida implementado entre la población de estudio.

\section{Conclusiones}

A través del proceso investigativo se pudo inferir que como estrategia de enseñanza la implementación de estrategias tecnológicas potencializa la atención de los estudiantes y permite que estos tomen un papel activo a fin de fortalecer y dinamizar la enseñanza. Se requerido que los docentes de la institución desarrollen una mejor cualificación en el uso de las herramientas de la información y la comunicación desde las tecnologías (Montenegro, Blanco y Cortés, 2013), (Cortés-Peña, Pinto-Santos y Atrio, 2015).

Los eventos de apropiación social del conocimiento, se describen como una experiencia para la presente investigación desde las actividades participativas del evento "el maestro tiene la palabra", en la cual se tuvo la oportunidad de socializar la experiencia investigativa con la comunidad docente educativa a nivel inter institucional, permitiendo en este sentido un reforzamiento y retro alimentación del proceso; es importante resaltar que a nivel de la comunidad local se requiere adelantar sobre el proceso de propagación, el cual se realizará en corto tiempo.

La discusión debe evidenciar un análisis crítico de los resultados obtenidos y dar ideas para el desarrollo de nuevas investigaciones.

\section{Referencias}

Arroyo, M., Faz de los Santos, L., Gasca, G. y Orozco, R. (2010). Mejoramiento de la comprensión lectora basada en el aprendizaje colaborativo en la enseñanza media básica. Apertura, 2 (2), 36-47. Recuperado de http://www.udgvirtual. udg.mx/apertura/index.php/apertura/ article/view/140/157

Avendaño-Villa, I., Cortés-Peña, O. y Guerrero-Cuentas, H. (2015). Competencias sociales y tecnologías de la información y la comunicación como factores asociados al desempeño en estudiantes de básica primaria con experiencia de desplazamiento forzado. Diversitas: Perspectivas En Psicología, 11(1), 13-36.

Cabrera-Medina, J., Sánchez-Medina, I. y Rojas-Rojas, F. (2016). Uso de objetos virtuales de aprendizaje ovas como estrategia de enseñanza-aprendizaje inclusivo y complementario a los cursos teóricos-prácticos. Revista Educación en Ingeniería, 11(22), 4-12.

Clavijo, Y., Herrera, A., Rhenals, J., y Tordecilla, R. (2015). Implementación de un objeto virtual de aprendizaje (Ova) para apoyar el proceso educativo en los estudiantes de grado cuarto de primaria de la Institución Educativa Alfonso Spath Spath. [Tesis de pregrado]. Universidad Los Libertadores, Montería. Recuperado de http://repository.libertadores.edu.co/ handle/11371/947

Cortés-Peña O., Pinto-Santos A. y Atrio, S. (2015). E-portafolio como herramienta construccionista del aprendizaje activo en tecnología educativa. Revista Lasallista de Investigación,12(2),36-44.

Cubillos, D. (Octubre 15, 2009). Nacimiento y evolución de las telecomunicaciones [Mensaje en un blog]. Recuperado de https://sites.google.com/site/ticsyopal5/ assignments/homeworkforweekofoctober25th 
Escobar, F. (2016). El uso de las TIC como herramienta pedagógica para la motivación de los docentes en el proceso de aprendizaje y enseñanza en la asignatura de inglés. [Tesis de maestría]. Universidad Pontificia Bolivariana, Medellín. Recuperado de https://repository.upb. e d u . c o / bi t s t r e a m/ h a nd. le/20.500.11912/2762/TESIS__MTIC_ FLOR_ESCOBAR\%20-\%20FINAL\%20 $\% 281 \% 29$.pdf?sequence $=1$

Fandos, M. (2003). Formación basada en las Tecnologías de la Información y Comunicación: Análisis didáctico del proceso de enseñanza-aprendizaje [Tesis doctoral]. Universitat Rovira I Virgili. Tarragona. Recuperado de http://www. tdx.cat/bitstream/handle/10803/8909/ Etesis 1.pdf

Hernández , R, Mendoza (2010). Metodología de la investigación. Quinta edición. México

Herrera, B. (2016). Cultura ciudadana y las tecnologías de la información y la comunicación. Barranquilla: Yoyobiz.

Martínez, M. y Badillo, A. (2013). Estrategias para el fortalecimiento de las TIC en las instituciones educativas oficiales de Colombia. Barranquilla: Colombia digital.
Montenegro, M., Blanco, P. y Cortés, O. (2013). Papel de las habilidades metalingüísticas en los procesos de lectura y escritura en la educación superior. Escenarios, 11(2), 82-86.

Padilla- Beltrán, J., Vega-Rojas, P. y Rincón-Caballero, D. (2014). Tendencias y dificultades para el uso de las TIC en educación superior. Entramado, 19, 272-295.

Parra, S., Gómez, M. y Pintor, M. (2014). Factores que inciden en la implementación de las TIC en los procesos de enseñanza-aprendizaje en 50 de Primaria en Colombia.Revista Complutense de Educación, 26 Núm. Especial, 197-213.

PoliVirtual in Colombia (2016). Aprendizaje online para los más pequeños. Google Chrome. Etiquetas Herramientas digitales. Recuperado de http://po$\begin{array}{lllllllllllll}\mathrm{l} & \mathrm{i} & \mathrm{v} & \mathrm{i} & \mathrm{r} & \mathrm{t} & \mathrm{u} & \mathrm{a} & \mathrm{l} & . & \mathrm{c} & \mathrm{o} & /\end{array}$ que-es-un-ova-y-cual-es-su-importancial

UNESCO. (2008). Estándares de competencia en TIC para docentes. París: Unesco.

Vélaz, C. y Vaillant, D. (2009). Aprendizaje y desarrollo profesional docente. España: Fundación Santillana. 